\title{
Pediatric Non-COVID-19 Community-Acquired Pneumonia in COVID-I9 Pandemic
}

\author{
Chienhsiu Huang (D) \\ Department of Internal Medicine, \\ Division of Chest Medicine, Dalin Tzu \\ Chi Hospital, Buddhist Tzu Chi Medical \\ Foundation, Dalin Town, Chiayi County, \\ Taiwan
}

Introduction: The COVID-19 lockdown strategies were associated with a significant decrease in infectious diseases disseminated through airborne or fecal-oral transmissions. Social distancing and other lockdown strategies effectively slowed down the spread of common respiratory viral diseases and decreased the need for hospitalization among children. Thus, a decline in the incidence of respiratory viral diseases had been reported following the COVID-19 outbreak. However, the trend of non-COVID-19 pneumonia in children remains uncertain. This study aimed to assess the impact of the COVID-19 pandemic on the incidence of non-COVID-19 community-acquired pneumonia in children.

Materials and Methods: We conducted a retrospective analysis of all patients (age $\leq 18$ years) presenting with pulmonary infection from January 2019 to December 2020 in a tertiary-level teaching hospital. We compared the number of pediatric patients admitted to our hospital with community-acquired pneumonia from January to December 2020 to those from the same period in 2019.

Results: A total of 65 patients were diagnosed with community-acquired pneumonia in 2019 and 18 in 2020. The number of patients with community-acquired pneumonia in 2020 was lower than that in 2019 by 47 cases in 2020 . The most age distribution of pediatric patients with community-acquired pneumonia was $2-5$ years in 2019 , indicating the decreasing number of community-acquired pneumonia patients from 36 patients in 2019 to 3 in 2020 .

Conclusion: The number of pediatric with community-acquired pneumonia was lower than in the same period in 2019, which markedly decreased $(-72.3 \%)$ in 2020 . These interventions applied to control the COVID-19 pandemic were effective not only in substantial changes in the seasonal influenza activity but also in decreasing cases of pediatric community-acquired pneumonia.

Keywords: coronavirus disease 2019, community-acquired pneumonia, pediatric infections, pandemic

\section{Introduction}

The coronavirus disease 2019 (COVID-19) outbreak was first detected in December 2019 in Wuhan, Hubei Province, China. In just 3 months, COVID-19 had quickly spread worldwide. This disease outbreak, COVID-19, then substantially worsened and was declared a pandemic by the World Health Organisation on March 11, 2020. Governmental agencies of different countries worldwide have been widely promoting several measures to prevent the COVID-19 outbreak, such as education on hand hygiene and cough etiquette, staying at home with respiratory symptoms, mask use in public places, social distancing and travel restrictions, and closure of schools, among others. ${ }^{1-5}$ The COVID-19 lockdown
Correspondence: Chienhsiu Huang Department of Internal Medicine, Division of Chest Medicine, Dalin Tzu Chi Hospital, Buddhist Tzu Chi Medical Foundation, NO. 2, Min-Sheng Road, Dalin Town, Chiayi County, Taiwan Tel +886-9-21552418

Email hgssport@yahoo.com.tw 
strategies were associated with a significant decrease in infectious diseases disseminated through airborne or faecal-oral transmissions. ${ }^{6}$ Social distancing and other lockdown strategies effectively slowed down the spread of common respiratory viral diseases and decreased the need for hospitalisation among children in Finland. ${ }^{7}$ Winter surveillance data from Japan has also demonstrated a decrease in seasonal influenza activity as compared to previous seasons. ${ }^{8}$ Thus, a decline in the incidence of respiratory viral diseases had been reported following the COVID-19 outbreak. Childhood community-acquired pneumonia (CAP) is one of the leading causes of childhood morbidity and mortality. Childhood communityacquired pneumonia is responsible for one-fifth of children's deaths around the world. ${ }^{9}$ According to World Health Organization, pneumonia accounts for $15 \%$ of all deaths of children under 5 years old in 2017 (http://www. who.int/mediacentre/factsheets/fs331/en/). The annual incidence of community-acquired pneumonia hospitalization was $15.7 / 10,000$ children $<18$ years old in US. ${ }^{10}$ The annual incidence of hospitalization for communityacquired pneumonia in children under 18 years in Taiwan was 69.5 cases per 100,000 population. ${ }^{11}$ However, the trend of non-COVID-19 communityacquired pneumonia in children remain uncertain in COVID-19 pandemic. This study aimed to assess the impact of the COVID-19 pandemic on the incidence of non-COVID-19 community-acquired pneumonia in children.

\section{Materials and Methods}

\section{Study Design}

Hospital Details

The Dalin Tzu Chi Hospital is a tertiary-level teaching hospital with 600 acute care beds (including 9 beds of pediatric beds) and an intensive care unit containing 59 beds (including 5 beds of pediatric intensive care unit).

\section{Patient Details}

We conducted a retrospective analysis of all patients (age $\leq 18$ years) presenting with pulmonary infection who were admitted to Dalin Tzu Chi Hospital from January 2019 to December 2020. We compared the number of pediatric patients admitted to our hospital with communityacquired pneumonia from January to December 2020 to those from the same period in 2019. Patients were diagnosed with pulmonary infection if their clinical symptoms and signs indicated infection and their chest radiology displayed a new acute pulmonary infiltration. Communityacquired pneumonia was defined as a pulmonary parenchymal infection in patients who acquire the condition in the community. ${ }^{12,13}$ The limited possibility of obtaining sputum in children are the barrier to obtaining adequate respiratory specimens for culture in children. Routine sputum culture for microbiological investigations was not checked for patients in our study. Routine Specimens for BinaxNOW urinary Streptococcus pneumoniae antigen detection test and Mycoplasma pneumoniae IgM test were obtained as part of the study protocol. Mycoplasma pneumoniae infection was confirmed with seropositive of Mycoplasma pneumoniae IgM in the acute stage. A positive urine Streptococcus pneumoniae antigen test in children with community-acquired pneumonia was considered a probable case of Streptococcus pneumoniae pneumonia. Neither culture nor real-time Polymerase Chain Reaction was performed to detect viral infections in our patients. All pneumonia patients of 2020 were screened for COVID-19 using a real-time reverse transcriptase-polymerase chain reaction test since Jan 22, 2020 (https://covid19.mohw.gov.tw/ch/cp-4842-53637$\underline{205 . h t m l)}$.

\section{Statistical Analysis}

Continuous variables were expressed as mean \pm standard deviation or median (range), whereas categorical variables were expressed as frequencies and percentages. The trend in change of pneumonia cases was analysed by linear-bylinear association using the chi-square test. All statistical analyses were conducted using the statistical package SPSS for Windows (Version 17.0, SPSS, Inc., Chicago, IL, USA). A $p$-value of $<0.05$ was considered statistically significant.

\section{Results}

A total of 83 children with community-acquired pneumonia were enrolled during the study period, including 65 cases in 2019 and 18 cases in 2020. Their age ranged from 7 weeks to 17.2 years with a median of 4.1 years. Of these, $17(20.5 \%)$ were under 2 years of age, $39(47.0 \%)$ were between 2 and 5 years, and $27(32.5 \%)$ were above 5 years. Tests for COVID-19 were negative for all community-acquired pneumonia patients in 2020 . We divide patients based on two periods, the first half of the year (January to June) and the second half of the year (July to December) as shown in Table 1. The number of patients with community-acquired pneumonia in 2020 was lower 
Table I Pediatric Non-COVID-19 Community-Acquired Pneumonia Patients Requiring Hospitalization, by Month and Age Cohorts

\begin{tabular}{|c|c|c|c|}
\hline & $\begin{array}{c}\text { Patients } \\
\text { No (\%) } \\
(2019)\end{array}$ & $\begin{array}{c}\text { Patients } \\
\text { No (\%) } \\
(2020)\end{array}$ & $\begin{array}{c}\text { Patients No } \\
(2019-2020) / \\
2019\end{array}$ \\
\hline $\begin{array}{l}\text { Month* } \\
\text { January to June } \\
\text { July to December }\end{array}$ & $\begin{array}{l}17(26.2 \%) \\
48(73.8 \%)\end{array}$ & $\begin{array}{c}10(55.6 \%) \\
8(44.4 \%)\end{array}$ & $\begin{array}{c}7 \text { (4I.2\%) } \\
40 \text { (83.3\%) }\end{array}$ \\
\hline $\begin{array}{l}\text { Age cohorts } \\
\text { Less than } 2 \text { years } \\
2-5 \text { years old } \\
6-18 \text { years old }\end{array}$ & $\begin{array}{l}\text { II (16.9\%) } \\
36(55.4 \%) \\
18(27.7 \%)\end{array}$ & $\begin{array}{l}6(33.3 \%) \\
3(16.7 \%) \\
9(50.0 \%)\end{array}$ & $\begin{array}{c}5(45.4 \%) \\
33(91.7 \%) \\
9(50 \% \%)\end{array}$ \\
\hline
\end{tabular}

Notes: *The decreasing number of patients with community-acquired pneumonia between 2019 and 2020 increased with time, from $-41.2 \%$ in the first half to $-83.3 \%$ in the second half of the year $2020(P=0.002)$.

Abbreviations: No, number; COVID-19, coronavirus disease 2019.

than that in 2019 by 47 cases $(-72.3 \%)$ in 2020 . The decreasing number of patients with community-acquired pneumonia between 2019 and 2020 increased with time, from $-41.2 \%$ in the first half to $-83.3 \%$ in the second half of the year $2020(\mathrm{P}=0.002)$. The study explored the influence of increasing age on the number of patients with community-acquired pneumonia by comparing the following three age cohorts: $<2,2-5$, and $>5$ years, during 2 years as shown in Table 1. The most age distribution of pediatric patients with community-acquired pneumonia was $2-5$ years in 2019 , indicating the decreasing number of community-acquired pneumonia patients from 36 patients in 2019 to $3(-91.7 \%)$ in 2020. A total of 31 pediatric patients were diagnosed with communityacquired pneumonia in 2019 with serology tests of community-acquired pneumonia pathogens, and all of whom had Mycoplasma pneumoniae. Twelve pediatric patients were diagnosed with community-acquired pneumonia in 2020 with serology tests of community-acquired pneumonia pathogens, including 11 with Mycoplasma pneumoniae and one with mixed infection with Streptococcus pneumoniae and Mycoplasma pneumoniae.

\section{Discussion}

The government of Taiwan took several rapid responses since January 2000 to prevent the COVID-19 outbreak, such as border control, case identification, quarantine of suspicious cases, and travel restriction, among others. The Taiwan Centres for Disease Control announced social distancing measures to encourage the general public to maintain social etiquette and to cancel conferences and social gatherings. The rules specified separate social distancing standards for restaurants, school campuses, offices, mass transport, supermarkets, and special institutions such as long-term care facilities and prisons. Mask was recommended to use widely throughout the country in all instances of close person-to-person contact. ${ }^{14}$ Students must wear masks all time and wash hands frequently at the school. Besides, the implementation period of government expense influenza vaccination is from October 2019 in Taiwan. The total number of outpatient and emergency room visits for influenza-like illness decreases since February 2020, which continued to decrease each week. Seasonal influenza activity continued to reduce to its lowest point in April 2020. The reduction in viral diseases may be attributed to COVID-19 preventive measures. Yamamoto et al reported that the number of patients with community-acquired pneumonia began to decrease in February 2020, demonstrating as significantly lower than those from the same period in the last 3 years. The author thought these COVID-19 outbreak measures might have indirectly contributed to a decreased number of cases through the prevention of common viral infections that could be a trigger of community-acquired pneumonia. ${ }^{15}$ However, no study has investigated the impact of preventive infection control measures in reducing the incidence of pediatric community-acquired pneumonia. Our study showed that the number of pediatric patients with community-acquired pneumonia reduced by an average of $72.3 \%$ in 1 year. The longer the number of days, the more the number of patients will decrease. The strategy to prevent the outbreak of COVID-19 has also been very effective in promoting protection against the pediatric communityacquired pneumonia.

Mycoplasma pneumoniae is an important causative pathogen of community-acquired pneumonia in children. The majority of the detected pneumonia pathogens were Mycoplasma pneumoniae in our study. Serology is an important tool for the diagnosis of Mycoplasma pneumoniae infection. The predominant role of serology in routine diagnosis is partly due to the ease of specimen collection and the widespread availability of serologic tests. To achieve a rapid diagnosis, elevated IgM antibodies represent a reliable indicator of Mycoplasma pneumoniae infection in children. IgM antibodies appear during the first week of the illness and reach peak titers during the third week. ${ }^{16}$ Measurement of IgM has been shown to be useful in pediatric Mycoplasma pneumoniae patients. ${ }^{17}$ 
Streptococcus pneumoniae is the leading causative pathogen of community-acquired pneumonia in children. In terms of community-acquired pneumonia pathogens, the incidence of patients infected with Streptococcus pneumoniae in our study is very low, which may be related to the following three factors. First, Streptococcus pneumoniae infections were diagnosed via urinary antigen tests, but the specificity of the urinary Binax NOW assay in the diagnosis of Streptococcus pneumoniae pneumonia was variable. ${ }^{18}$ The use of a positive urine antigen test as a diagnosis of Streptococcus pneumoniae infection may be under diagnosis in our series. Second, the incidence of community-acquired pneumonia and bacterial pneumonia burden in the pediatric population has significantly decreased worldwide in the past decades, because of the increasingly widespread use of effective means of prevention such as Haemophilus influenzae type $b$ vaccine and Streptococcus pneumoniae conjugate vaccines $(\mathrm{PCVs}){ }^{10,19-21}$ In Taiwan, the 7-valent Streptococcus pneumoniae conjugate vaccine (PCV7) and the 13-valent Streptococcus pneumoniae conjugate vaccine (PCV13) were introduced in 2005 and 2011, respectively. Within one year of optional PCV13 immunization, the annual CAP hospitalization rates had lowered in children under 5 years of age. Since 2013, all children aged 2-5 years and since 2014, all children aged 1-5 years were provided with PCV13 via a catch-up immunization program by the national immunization program. The annual incidence rate of Streptococcus pneumoniae pneumonia is expected to continue falling under the current PCV13 immunization policy. ${ }^{11,22}$ Third, McCullers et al showed that influenza infections increase the risk for developing secondary bacterial disease, particularly with streptococcal pneumonia. ${ }^{23}$ Weinberger et al reported a significant increase in pneumococcal hospitalizations that corresponded to the timing of the highest pandemic influenza activity. ${ }^{24}$ A significant decrease in pediatic Streptococcus pneumoniae pneumonia hospitalizations in our study corresponded to the lowest influenza activity during the COVID-19 pandemic.

\section{Psychosocial Challenges for Young People and Children of School Closure in COVID-I 9 Pandemic}

Community infection has occurred during the COVID-19 epidemic in Taiwan. The Ministry of Education announced the suspension of classes across the country beginning in May 19, 2021. Teenagers and children may have initially enjoyed not having to go to school, but school closures are likely to have many unintended and potentially serious consequences on the psychological and physical health of children and young people. All students must stay at home, social interaction is curtailed and the mental health of teenagers and children may face challenges. Schools play a key role in providing support to teenagers and children. Schools provide teenagers and children with a safe daily space, allowing them to talk to trusted adults, and allowing them to be face-to-face with friends in a safe place. Teachers not only teach students curriculum, they also teach the students how to get along with others, cultivate empathy, and how to play peacefully with each other at school. Although schools reopened on September 1, 2021, students must still keep social distancing at school. They still cannot be close to their friends during rest and play. It is difficult for teenagers to enforce social distancing regulations in schools and it is very bad for the children's mental health. Teachers need to understand children's feelings, not just focus on their lessons. Teachers and parents should not put pressure on their children and it will be more helpful to help them get out of isolation. In order for children to learn, they need to be given some time to adjust their emotions before they can start normal learning.

\section{Telemedicine in Taiwan}

Taiwan is a heavily populated country comprised of a main island where three-fourths of the land area is mountainous as well as several smaller outlying islands. In rural, mountainous, and isolated areas, medical resources are under distributed. Telemedicine provides a convenient and spaceless measure for distributing medical education and remote care to these locations. The development of telemedicine in Taiwan began under the National Information Infrastructure (NII) project and the NII Steering Committee was installed in August 1994. Taiwan's medical law restricts telemedicine to mountainous regions, outlying islands, and remote areas. The COVID-19 pandemic has rapidly transformed health care systems worldwide. The COVID-19 pandemic and various lockdown measures hindered face-to-face healthcare delivery. Telemedicine may play a vital role in timely delivery of care and protect both patients and healthcare workers from exposure. ${ }^{25,26}$ The Taiwanese government has ensured that administrative and legal structures are in place to make telemedicine accessible. Medical institutions can provide telemedicine services for individuals under home quarantine during the 
COVID-19 pandemic (https://www.nhi.gov.tw/Law Detail.aspx? n=5597495EEC 8219A $1 \& \mathrm{sms}=$ 36A0BB334ECB4011\&s=09E0067E0D01063D).

Telemedicine is an effective instrument for monitoring COVID-19 infection in quarantined travelers. ${ }^{27}$ However, many physicians are unwilling to participate in telemedicine and many specialists prefer face-to-face visits, which is a problem encountered in promoting telemedicine in Taiwan.

\section{Study Limitations}

Our report is a small single-unit study with few participants. These results must be interpreted with caution, especially the results of community-acquired pneumonia pathogens. It is impossible to make any firm conclusions based on this preliminary result. There were some limitations in this retrospective study. First, our study was conducted in a single tertiary teaching hospital and the results may therefore not be generalizable due to different patient populations in other hospitals and countries. Second, for patients aged $<18$ years, sufficient sputum culture tests were not performed in comparison with blood culture and serological tests. The use of a single antigen for the serological diagnosis of pneumococcal pneumonia may be limitation. We may underdiagnose pneumococcal and other bacterial pneumonia. The use of reverse transcriptase-polymerase chain reaction test to check bacterial pneumonia could be a useful additional diagnostic method. Third, we used a single Mycoplasma pneumoniae $\operatorname{IgM}$ titer, the prevalence of Mycoplasma could be overestimated. To consolidate our findings, a national multicenter prospective further study should include a greater number of patients and focus on the prevalence and pathogens of pediatric non-COVID-19 community-acquired pneumonia in COVID-19 pandemic.

\section{Conclusions}

The number of pediatric community-acquired pneumonia was lower than in the same period in 2019, which markedly decreased $(-72.3 \%)$ in 2020 . These interventions applied to control the COVID-19 pandemic were effective not only in substantial changes in the seasonal influenza activity but also in decreasing cases of pediatric community-acquired pneumonia.

\section{Data Sharing Statement}

The datasets generated during and/or analyzed during the current study are available from the corresponding author on reasonable request.

\section{Compliance with Ethics Guidelines}

The study conformed to the Declaration of Helsinki 1975, revised Hong Kong 1989. The project was approved by the Buddhist Dalin Tzu Chi General Hospital Research Ethics Committee (Approved IRB No.: B11002015)

Patient parental consent to review their medical records was not required. The reasons for the waiver were as below:

1.This study was not a prospective clinical trial and was a retrospective medical record data collection and analysis.

2. This study was the lowest risk, and the possible risk to the research subject did not exceed the nonparticipating researchers, and the exemption from prior consent did not affect the rights of the researcher.

3. Exempt informed consent did not affect the subject rights and wellbeing. Research could not be performed without exempting informed consent.

4. Appropriate privacy protection measures are in place to protect children participating in the research.

Appropriate privacy protection measures are in place to protect children participating in the research as follows:

1.This is a retrospective observational study and the retrospective medical records do not involve the collection and use of specimens.

2.During data collection, the subjects' identities are via the hospital inpatient information system.

3. We check all subjects' CXR findings and discharge summary. We do not collect these data.

4. We do not collect the name, gender, history number and other personal information of all subjects in this study.

5 . We collect the total number of pneumonia cases by the months of 2019 and 2020 only. We do not collect the detailed hospital course of all pneumonia cases.

6 . We collect the age distribution of all pneumonia cases only. We do not collect the actual age of all subjects.

7. We collect the total cases of positive results of laboratory related to pneumonia pathogens (such as Mycoplasma pneumoniae IgM, Streptococcus pneumoniae antigen test, and bacterial culture) by the year of 2019 and 2020 only. We do not collect which subjects showed positive results of laboratory related to pneumonia pathogens.

8.All pneumonia patients of 2020 were screened for COVID-19 using a real-time reverse transcriptase- 
polymerase chain reaction test since Jan 22, 2020 in our hospital and we did not collect these data of all subjects in this study.

9.The legal biological database cannot identify a specific individual. It cannot identify a particular individual's data, files, documents, information, or samples for research. Never involve disclosure of the subjects' personal privacy and basic information at any time.

10.After the research is completed, all relevant data of the subjects participating in the research will be destroyed according to law.

\section{Author Contributions}

The author made substantial contributions to conception and design, acquisition of data, or analysis and interpretation of data; took part in drafting the article or revising it critically for important intellectual content; agreed to submit to the current journal; gave final approval of the version to be published; and agree to be accountable for all aspects of the work.

\section{Funding}

There was no funding in this study.

\section{Disclosure}

The author declares no competing interests.

\section{References}

1. Ghanchi A. Adaptation of the national plan for the prevention and fight against pandemic influenza to the 2020 COVID-19 epidemic in France. Disaster Med Public Health Prep. 2020;7:1-3.

2. Macartney K, Quinn HE, Pillsbury AJ, et al. Transmission of SARS-CoV-2 in Australian educational settings: a prospective cohort study. Lancet Child Adolesc Health. 2020;4:807-816. doi:10.1016/ S2352-4642(20)30251-0

3. Mensah AA, Sinnathamby M, Zaidi A, et al. SARS-CoV-2 infections in children following the full re-opening of schools and the impact of national lockdown: prospective, national observational cohort surveillance, July-December 2020, England. $J$ Infect. 2021;82:67-74. doi:10.1016/j.jinf.2021.02.022

4. Panovska-Griffiths J, Kerr CC, Stuart RM, et al. Determining the optimal strategy for reopening schools, the impact of test and trace interventions, and the risk of occurrence of a second COVID-19 epidemic wave in the UK: a modelling study. Lancet Child Adolesc Health. 2020;4:817-827. doi:10.1016/S2352-4642(20)30250-9

5. Kruizinga MD, Peeters D, van Veen M, et al. The impact of lockdown on pediatric ED visits and hospital admissions during the COVID19 pandemic: a multicenter analysis and review of the literature. Eur $J$ Pediatr. 2021;180:2271-2279. doi:10.1007/s00431-021-04015-0

6. Angoulvant F, Ouldali N, Yang DD, et al. COVID-19 pandemic: impact caused by school closure and national lockdown on pediatric visits and admissions for viral and non-viral infections, a time series analysis. Clin Infect Dis. 2021;72:319-322. doi:10.1093/cid/ ciaa 710
7. Kuitunen I, Artama M, Mäkelä L, Backman K, Heiskanen-Kosma T, Renko M. Effect of social distancing due to the COVID-19 pandemic on the incidence of viral respiratory tract infections in children in finland during early 2020. Pediatr Infect Dis J. 2020;39:e423-e427. doi:10.1097/INF.0000000000002845

8. Sakamoto H, Ishikane M, Ueda P. Seasonal influenza activity during the SARSCoV- 2 outbreak in Japan. JAMA. 2020;323:1969-1971. doi:10.1001/jama.2020.6173

9. Williams BG, Gouws E, Boschi-Pinto C, Bryce J, Dye C. Estimates of world-wide distribution of child deaths from acute respiratory infections. Lancet Infect Dis. 2002;2:25-32. doi:10.1016/S14733099(01)00170-0

10. Jain S, Williams DJ, Arnold SR, et al. Community-acquired pneumonia requiring hospitalization among U.S. Children $N$ Engl J Med. 2015;26(372):835-845. doi:10.1056/NEJMoa1405870

11. Chi H, Huang YC, Liu CC, et al. Characteristics and etiology of hospitalized pediatric community-acquired pneumonia in Taiwan. J Formos Med Assoc. 2020;119:1490-1499. doi:10.1016/j.jfma.20 20.07 .014

12. Harris M, Clark J, Coote N, et al; British Thoracic Society Standards of Care Committee. British Thoracic Society guidelines for the management of community acquired pneumonia in children: update 2011. Thorax. 2011;66:ii1-ii23. doi:10.1136/thoraxjnl-2011200598

13. Bradley JS, Byington CL, Shah SS, et al. The management of community-acquired pneumonia in infants and children older than 3 months of age: clinical practice guidelines by the Pediatric Infectious Diseases Society and the Infectious Diseases Society of America. Clin Infect Dis. 2011;53:e25- e76. doi:10.1093/cid/cir531

14. Wang CJ, Ng CY, Brook RH. Response to COVID-19 in Taiwan: big data analytics, new technology, and proactive testing. JAMA. 2020;323:1341-1342. doi:10.1001/jama.2020.3151

15. Yamamoto T, Komiya K, Fujita N, Okabe E, Hiramatsu K, Kadota JI. COVID-19 pandemic and the incidence of community-acquired community-acquired pneumonia in elderly people. Respir Investig. 2020;18(58):435-436. doi:10.1016/j.resinv.2020.09.001

16. Jacobs E, Bennewitz A, Bredt W. Reaction pattern of human anti-Mycoplasma pneumoniae antibodies in enzyme-linked immunosorbent assays and immu- noblotting. J Clin Microbiol. 1986;23:517-522. doi:10.1128/jcm.23.3.517-522.1986

17. Waris ME, Toikka P, Saarinen T, et al. Diagnosis of Mycoplasma pneumoniae pneumonia in children. $J$ Clin Microbiol. 1998;36:3155-3159. doi:10.1128/JCM.36.11.3155-3159.1998

18. Shen CF, Wang SM, Liu CC. A new urinary antigen test score correlates with severity of pneumococcal pneumonia in children. $J$ Formos Med Assoc. 2011;110:613-618. doi:10.1016/j. jfma.2011.08.002

19. Esposito S, Principi N. Pneumococcal vaccines and the prevention of community-acquired pneumonia. Pulm Pharmacol Ther. 2015;32:124-129. doi:10.1016/j.pupt.2014.02.003

20. Peltola H. Worldwide Haemophilus influenzae type $b$ disease at the beginning of the 21st century: global analysis of the disease burden 25 years after the use of the polysaccharide vaccine and a decade after the advent of conjugates. Clin Microbiol Rev. 2000;13:302-317. doi:10.1128/CMR.13.2.302

21. Wahl B, O'Brien KL, Greenbaum A, et al. Burden of Streptococcus pneumoniae and Haemophilus influenzae type $\mathrm{b}$ disease in children in the era of conjugate vaccines: global, regional, and national estimates for 2000-15. Lancet Glob Health. 2018;6:e744-757. doi:10.1016/ S2214-109X(18)30247-X

22. Su WJ, Lo HY, Chang $\mathrm{CH}$, Chang LY, Chiu CH, Lee PI. Effectiveness of pneumococcal conjugate vaccines of different valences against invasive pneumococcal disease among children in Taiwan: a nationwide study. Pediatr Infect Dis J. 2016;35:e124-e133. doi:10.1097/INF.0000000000001054 
23. McCullers JA. Insights into the interaction between influenza virus and pneumococcus. Clin Microbiol Rev. 2006;19:571-582. doi:10.1128/CMR.00058-05

24. Weinberger DM, Simonsen L, Jordan R, Steiner C, Miller M, Viboud C. Impact of the 2009 influenza pandemic on pneumococcal pneumonia hospitalizations in the States. $J$ Infect Dis. 2012;205:458-465. doi:10.1093/infdis/jir749

25. Greenhalgh T, Koh GCH, Car J. Covid-19: a remote assessment in primary care. BMJ. 2020;368:m1182. doi:10.1136/bmj.m1182
26. Hollander JE, Carr BG. Virtually Perfect? Telemedicine for Covid-19. $N$ Engl J Med. 2020;382:1679-1681. doi:10.1056/ NEJMp2003539

27. Yen YF, Tsai YF, Su VY, et al. Use and cost-effectiveness of a telehealth service at a centralized COVID-19 quarantine center in Taiwan: cohort Study. J Med Internet Res. 2020;22(12):e22703. doi: $10.2196 / 22703$

\section{Publish your work in this journal}

The International Journal of General Medicine is an international, peer-reviewed open-access journal that focuses on general and internal medicine, pathogenesis, epidemiology, diagnosis, monitoring and treatment protocols. The journal is characterized by the rapid reporting of reviews, original research and clinical studies across all disease areas. The manuscript management system is completely online and includes a very quick and fair peer-review system, which is all easy to use. Visit http://www.dovepress.com/ testimonials.php to read real quotes from published authors.

Submit your manuscript here: https://www.dovepress.com/international-journal-of-general-medicine-journal 